\title{
Miranda
}

Revue pluridisciplinaire du monde anglophone /

Multidisciplinary peer-reviewed journal on the English-

speaking world

$21 \mid 2020$

Modernism and the Obscene

\section{Isabelle Gaudy-Campbell, Céline Horgues (eds), Linguistique anglaise et oralité : vers une approche intégrée}

\section{Saandia Ali}

\section{OpenEdition}

\section{Journals}

Édition électronique

URL : http://journals.openedition.org/miranda/29443

DOI : $10.4000 /$ miranda.29443

ISSN : 2108-6559

\section{Éditeur}

Université Toulouse - Jean Jaurès

\section{Référence électronique}

Saandia Ali, «Isabelle Gaudy-Campbell, Céline Horgues (eds), Linguistique anglaise et oralité : vers une approche intégrée », Miranda [En ligne], 21 | 2020, mis en ligne le 13 octobre 2020, consulté le 16 février 2021. URL : http://journals.openedition.org/miranda/29443; DOI : https://doi.org/10.4000/miranda. 29443

Ce document a été généré automatiquement le 16 février 2021.

\section{cc) (1)}

Miranda is licensed under a Creative Commons Attribution-NonCommercial-NoDerivatives 4.0

International License. 


\section{Isabelle Gaudy-Campbell, Céline Horgues (eds), Linguistique anglaise et oralité : vers une approche intégrée}

Saandia Ali

\section{RÉFÉRENCE}

Isabelle Gaudy-Campbell, Céline Horgues (eds) : Linguistique anglaise et oralité : vers une approche intégrée (Paris : Presses Sorbonne Nouvelle, 2019), 180 p. ISBN :

978-2-37906-017-5. 
Isabelle Gaudy-Campbell et Céline Horgues

L I N G U G T I Q UE

\section{A N GLA I SE E T ORALITE}

VERS UNE APPROCHE INTEGREE

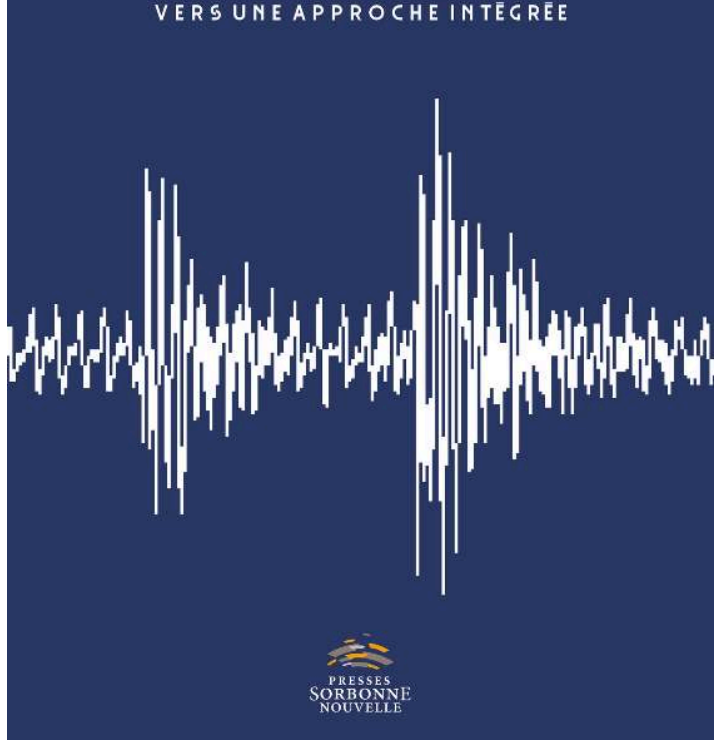

1 L'ouvrage collectif d'Isabelle Gaudy-Campbell et de Céline Horgues affiche d'emblée par son titre l'objectif ambitieux de faire dialoguer et interagir différents champs disciplinaires de la linguistique afin de proposer une " approche intégrée » de l'étude de la langue orale. Au fil des contributions variées, les auteurs font appel à différents outils, corpus, méthodologies et branches de la linguistique pour analyser l'oralité en langue anglaise. Il ne s'agit pas de faire de « l'art pour l'art », de la phonétique pour la phonétique, ou de «l'analyse sonore pour elle-même », mais de la phonétique et de la phonologie en relation avec le sens, le discours et la communication. La phonétique n'est pas non plus uniquement au service de la langue qu'elle viendrait illustrer, conforter ou accompagner mais elle s'intègre pleinement à la création du sens du message. Le choix des termes conceptuels utilisés par les auteurs pour y faire référence est très varié tout au long de l'ouvrage et reflète la complexité de l'objet d'étude ainsi que les différents points de vue, les multiples facteurs et niveaux d'analyse que l'on peut invoquer pour éclairer le fonctionnement de l'oral.

2 L'ouvrage se compose de trois parties principales elles-mêmes divisées en plusieurs chapitres : la première partie contient deux chapitres, la seconde quatre chapitres et la troisième trois chapitres. Un avant-propos et une postface jalonnent l'ouvrage.

L'avant-propos permet de décrire la genèse de ce travail d'écriture et de recherche qui vient consacrer les travaux réalisés par un ensemble de chercheurs dans les douze dernières années au sein du réseau informel OSLIA (Oral Spontané et Linguistique Anglaise). L'ouvrage est aussi un hommage aux recherches menées par claude Charreyre, membre de ce groupe de recherche et récemment décédée. Même si celle-ci ne se considérait pas comme une oraliste dont l'objet de recherche aurait été la langue orale essentiellement, ses recherches ont toujours pris en compte les marqueurs oraux. Dans ses travaux, et ce dès 1982, elle met déjà en valeur le rôle crucial de la prosodie dans toute analyse grammaticale linguistique. Les objectifs et les défis que pose la 
réalisation d'un tel ouvrage sont mis en valeur dans cet avant-propos. Il s'agit de travailler à une meilleure compréhension et une meilleure appréhension du fonctionnement de la langue orale spontanée. Il faudra alors fournir des clés, des outils et des approches méthodologiques permettant d'analyser l'oral comme faisant partie intégrante de la communication et de la création du sens. Quelques difficultés sont mentionnées telles que le caractère synchrone de l'oralité, la superposition des phénomènes linguistiques et phonétiques, la co-construction du sens en temps réel, les ratés potentiels et l'analyse post-conversation qui sera l'objet du travail du linguiste. La filiation avec les travaux d'Antoine Culioli $(1991,1999)$ par l'intermédiaire de Claude Charreyre est aussi posée à cette occasion.

\section{Linguistique de l'écrit et analyse de l'oral : filiation d'une démarche en linguistique énonciative}

4 La première partie de l'ouvrage collectif est construite autour de deux articles qui revisitent les travaux de Claude Charreyre. Le premier, "Pour une syntaxe énonciative : l'apport de Claude Charreyre ", rend hommage aux travaux de Claude Charreyre non publiés tels que son HDR. Le texte comprend des notes très personnelles, Alain Deschamps faisant référence à Claude Charreyre en utilisant uniquement son prénom. L'auteur présente de façon synthétique et claire les apports de Claude Charreyre sur le plan théorique. Son interprétation du cadre théorique Culiolien à travers de multiples exemples originaux et pertinents vise à étendre cette analyse à un domaine plus large passant de la syntaxe de la phrase à la syntaxe de l'énoncé dans toute sa complexité. Les notions de groupe nominal et de groupe verbal sont ensuite revisitées avant de présenter une vision et une théorie applicables à l'étude des anaphores. C'est une approche particulièrement originale qui ouvre la voie à l'analyse des anaphores verbales. Ce chapitre pose clairement son objectif qui est de faire connaître, même de façon jugée trop synthétique par l'auteur, la richesse du raisonnement de Claude Charreyre caractérisé par sa "grande maîtrise des outils théoriques énonciatifs », « un don pour trouver des exemples remarquables » et « une grande finesse dans les analyses de suites textuelles simples et complexes" (33). L'auteur fait le choix de se concentrer sur l'apport en linguistique de l'écrit et dans le domaine de la syntaxe plutôt que d'aborder les questions liées à l'oralité dans cet hommage.

5 Dans le second chapitre, «I et le question tag, ou le jeu de l'énonciation : un protocole d'analyse pour le traitement de l'oral », les co-auteurs Isabelle Gaudy-Campbell et Mark Gray s'embarquent dans une relecture d'un article de Claude Charreyre publié en 1984. Celui-ci a été riche d'enseignements pour les deux auteurs qui en tirent chacun dans leur domaine et dans leurs recherches respectives des leçons pertinentes et considérables. Mark Gray développe les liens entre la dimension énonciative et le contour intonatif dans les question tag pendant qu'Isabelle Gaudy-Campbell définit les contours d'un protocole d'analyse de l'oral s'inspirant de l'article de Charreyre (1984) et en propose une extension vers une approche interactive liant syntaxe, sémantique, énonciation et prosodie. La portée du question tag dans les énoncés à deux propositions est le point de départ de la recherche menée par Charreyre (1984) et Gaudy-Campbell et Gray (2019). Ils analysent plus précisément « les choix mélodiques effectués sur les reprises par question tag ». Les exemples fabriqués utilisés dans l'article de Charreyre 
(1984) sont complétés par des exemples tirés de corpus authentiques permettant une représentation statistique. Mark Gray montre que la montée intonative accompagnée avec une forme interrogative, indique que le locuteur se soumet au jugement de validité de son interlocuteur (38). Au contraire, lorsque le question tag est réalisé avec une chute mélodique, l'auteur identifie une confirmation liée à « une question qui se pose » (38). Le contour du question tag est aussi associé à la place de l'accent nucléaire qui va conditionner et contraindre les choix mélodiques possibles. S'appuyant sur les travaux de Gray et Nicaise (2002), les auteurs élaborent l'approche globale suivante de la contribution des mélodies et l'interprétation des énoncés.

Dans cette approche on considère que la contribution des mélodies à l'interprétation des énoncés appartient au domaine du repérage d'une relation prédicative par rapport à la situation d'énonciation. Le choix mélodique d'un énoncé permet notamment d'identifier comment la relation prédicative est repérée par rapport à l'énonciateur et au co-énonciateur. Dans le cas des mélodies descendantes, le repérage par rapport à l'énonciateur est privilégié, tandis que les mélodies montantes privilégient la prise en compte du co-énonciateur/ interlocuteur (40).

7 On passe ensuite du lien entre syntaxe et prosodie à une approche plus globale de l'apport de l'article phare. Isabelle Gaudy-Campbell explore ainsi la question de la linéarité de la production de la parole qui mène à considérer les phénomènes de reprise (ou question tag ici) comme "seconds" voire même "secondaires" et propose de s'affranchir de cette affirmation en s'appuyant sur les concepts de programmation et d'anticipation (mis en place dans Gaudy 2000) et sur les principes de la linguistique énonciative: la programmation émane de l'énonciateur, l'anticipation du coénonciateur (43).

De façon concrète, par exemple, le choix du contour mélodique associé à l'accent nucléaire dans la proposition principale interagit avec le contour mélodique appliqué au question tag pour véhiculer du sens. Se pose alors la question de la stratégie discursive en jeu au moment de l'énonciation. C'est dans cette interaction entre l'analyse des paramètres énonciatifs et celle des paramètres prosodiques (intonatifs ici) que l'auteur étudie le statut des énoncés. On retrouve l'idée d'un « dépassement » des limites de la micro-syntaxe pour aller vers l'étude de la macro-syntaxe. Comme Claude Charreyre auparavant, Isabelle Gaudy-Campbell passe de la phrase à l'énoncé, aux propositions enchainées et, au-delà de celles-ci, à l'étude du paragraphe oral.

9 L'approche développée par les auteurs «convoque tour à tour la prosodie, le sémantisme, les repérages énonciatifs et la syntaxe » (48). Elle met en lumière l'apport de l'oral dans la linguistique traditionnelle en en définissant des contours précis liés au " positionnement énonciatif » (49), aux écarts par rapport aux normes de la langue et " au trait sonore accompagné de sa dimension prosodique » (49). Le cadre théorique ainsi que la filiation de ce cadre sont mis en place dans cette première partie qui ouvre la voie aux recherches d'application qui vont suivre.

\section{Structuration et agencement discursif}

10 Dans cette seconde partie les chercheurs mettent en application une approche intégrée de l'étude de l'oral spontané en observant une série de marqueurs spécifiques à l'oral 
spontané tel que I mean et I think. L'analyse de l'utilisation de ces marqueurs oraux est réalisée à travers le prisme de la linguistique énonciative.

Jean Szlamowicz se penche sur "I mean comme ligateur flottant » à l'aide d'un corpus de travaux remontant à 2001 et d'un ensemble d'exemples tirés de son corpus de thèse (1997-1998). I mean est présenté comme un marqueur autonome qui n'appartient pas à la relation prédicative. Il est accompagné d'indices intonatifs discrets mais fondamentaux sur le plan de l'énonciation. Sont cités par exemple les pics d'intensité, les variations légères de la fréquence fondamentale et les variations de durée ayant un impact sur la saillance du marqueur. Celui-ci est défini comme un marqueur à la fois anaphorique et cataphorique permettant de préciser sa pensée, de reformuler sa pensée, de la clarifier ou d'en proposer des développements. Il est lié à des phénomènes égocentrés (centrés sur le locuteur lui-même) par opposition au marqueur you know par exemple qui est associé au co-énonciateur. L'auteur puise tour à tour dans la sémantique, dans la morpho syntaxe et dans l'analyse du discours et de son intonation pour révéler le statut discursif énonciatif de I mean. Des exemples précis sont ensuite analysés à partir de l'étude du corpus de thèse et en relation avec la valeur de rectification que peut porter I mean ainsi qu'avec la valeur d'explicitation.

12 Steven Schaefer développe une analyse combinatoire de la forme I think mêlant sémantique, syntaxe du discours et variations prosodiques. L'apport complexe des variations prosodiques à l'interprétation du sens est éclairé par une approche énonciative de l'analyse du corpus dialogique choisi (une interview entre un journaliste et Tony Blair). L'objectif principal est de mieux comprendre la contribution de I think en contexte et dans ses différentes formes : que celui-ci soit un verbe introductif, un parenthétique qui apporte un commentaire ou un marqueur discursif vide. L'article de Claude Charreyre de 1984 représente de nouveau une inspiration pour cette étude intégrant l'oral et surtout la dimension prosodique dans l'analyse d'un marqueur discursif. La théorie des opérations énonciatives (TOE) est au cœur de la recherche menée par l'auteur et souligne l'idée d'une prise en charge de l'énoncé par «l'énonciateur-origine» (73). L'effet de la prosodie ainsi que celui de la position linéaire sur des énoncés du corpus sont étudiés en détail. Le placement de l'accent nucléaire sur le pronom I ou bien sur le verbe think est pris en considération comme dans les recherches précédentes apparaissant dans la littérature. Cependant Steven Schaefer montre qu'il n'y a pas de corrélat direct entre prosodie et interprétation et que c'est bien « l'analyse combinatoire de I think en tant que prise en charge modale d'un énoncé » (82) qui permet d'en éclairer les usages.

13 Le chapitre $\mathrm{V}$, «Associer les pièces du puzzle : marqueurs verbaux et prosodiques en oral spontané ", écrit par Marine Riou, explore le marquage de la transition topicale par deux modalités dans le discours : les marqueurs de discours et le registre de voix étendue. Le travail de recherche s'appuie sur un corpus oral spontané en anglais américain datant des années 2000. Celui-ci est transcrit selon les conventions de l'analyse conversationnelle (voir Jefferson 2004). La pragmatique et l'analyse conversationnelle sont combinées afin d'étudier ce phénomène. Le corpus oral est utilisé pour détecter automatiquement les changements de registre coïncidant avec une transition topicale et pour réaliser des recherches de marqueurs discursifs associés pour en fournir une étude statistique. Les résultats montrent que le locuteur a véritablement le choix d'utiliser soit un marqueur discursif uniquement, soit un marqueur prosodique uniquement ou bien les deux afin de signaler le changement de 
topic. L'auteur favorise donc une approche combinatoire appliquée à la transition topicale qui analyse le rôle joué par les marqueurs dans l'interaction. Cette approche combinatoire permet de mettre en lumière la «synonymie pragmatique " (86) des marqueurs de la transition topicale (c'est-à-dire le fait que deux marqueurs puissent être utilisés à une même fin).

Yann Fuchs présente ensuite une étude expérimentale de l'usage des quotatifs say et be like en discours: "Quotatifs et performance multimodale: quelques pistes méthodologiques ». Cette étude vise à mettre en place une approche méthodologique multimodale pour l'observation de ces quotatifs dans un corpus conversationnel constitué par l'auteur (Cambridge Student Corpus 2010-2011). Pour la première fois dans cet ouvrage un auteur introduit la dimension gestuelle dans l'étude des phénomènes verbaux à l'oral. Il prend en compte le canal verbal, le canal sonore et le canal visuel afin d'interpréter la dimension orale des quotatifs. Le concept de performance multimodale est associé à la distribution fonctionnelle sur le plan sémantique, pragmatique et interactionnel. La chaîne segmentaire, les éléments prosodiques et les attitudes corporelles sont intégrés dans cette approche. Les quotatifs souvent considérés comme des "introducteurs de discours direct» (100), ont connu une évolution récente dynamique dans la langue anglaise, ce qui explique en partie l'intérêt que les chercheurs leur portent. L'auteur va donc tester les hypothèses les plus fréquentes émises au sujet de be like et say à l'aide de son corpus. Alors que say serait le plus souvent associé à une forme de neutralité représentée sur le plan sonore et niveau de la performance gestuelle, be like serait assimilé à une forme de "dramatisation du discours" accompagnée d'une performance à la fois sonore et gestuelle. Les premiers résultats confirment en partie ces hypothèses et mettent en valeur une certaine prééminence des variations de hauteur de voix sur les variations gestuelles co-verbales dans la distinction du comportement de say et be like. Les microanalyses appliquées à différents locuteurs montrent toutefois que le quotatif say est employé avec beaucoup plus de variabilité que le quotatif be like.

\section{Contribution de la prosodie à la grammaire de l'oral}

Les contributions de cette troisième partie s'éloignent quelque peu du cadre de la théorie des opérations énonciatives pour observer de façon globale et combinée la contribution des différents facteurs prosodiques à la structuration du discours oral. Sur le plan de la prosodie, la hauteur mélodique, les pauses et le débit sont observés de façon systématique recherchant leur impact sur la structuration du discours et leur contribution à la "construction du sens dans sa globalité » (117). Le chapitre VII, " Structurer le discours : le rôle de la prosodie » est consacré aux recherches de Susanne Moore Mauroux présentant l'étude d'un corpus d'émissions de radio qui comportent toutes un présentateur et des invités. Le genre défini est médiatique, dialogal et interactionnel. Il s'agit de discours de type très structuré comportant des phases précises et systématiques et l'auteur s'intéresse en particulier aux phases de présentation (pendant lesquelles le présentateur présente les invités) qu'elle met en opposition avec les phases d'interaction (pendant lesquelles les invités répondent à des questions). Dans chacune de ces phases, on explore l'utilisation des multiples facteurs prosodiques mesurés pour comprendre leur participation et la façon dont ils se combinent entre eux ou bien au contraire sont autonomes et dominants. L'observation 
de locuteurs spécifiques montre notamment une corrélation entre un débit lent et des pauses lors des phases présentatives et, d'autre part, un débit plus rapide dans les phases interactives qui sont plus spontanées. Des marqueurs lexicaux tels que les déictiques, et les connecteurs logiques sont associés à ces observations et en particulier aux variations de la fréquence fondamentale. L'auteur se penche aussi sur les changements thématiques en interaction avec le pitch reset qui semble systématique et la modulation du débit et des pauses. Cette partie fait écho aux travaux de Marine Riou qui présentait une analyse des transitions topicales.

Sophie Herment propose une étude des structures syntaxiques de focalisation non canoniques en discours dans le chapitre VIII intitulé : «L'interface syntaxe/prosodie : réinterprétation en discours oral et approche discursive de la prosodie ». En se basant sur des corpus oraux semi-spontanés (le corpus ICE-GB et le corpus Aix-Marsec), elle étudie les caractéristiques syntaxiques et prosodiques d'un ensemble de structures de focalisation telles que les clivées, les dislocations à droite ou le «do emphatique ». L'objectif de ce travail est la mise à jour d'une approche discursive combinatoire appliquée à la grammaire de l'oral. Un cadre théorique est bien défini mettant en relation d'une part la structure informationnelle des énoncés et d'autre part les éléments prosodiques de façon structurée. Sont pris en compte le découpage des unités intonatives, le placement du noyau et le type de contour mélodique associé. L'auteur réinterprète les relations entre la syntaxe et la prosodie en discours montrant notamment l'absence de contradiction entre "économie linguistique et redondance " (136) dans les cas où à la fois la syntaxe et la prosodie sont utilisées pour la mise en relief. Les écarts entre les patrons attendus et les patrons réalisés sont présentés comme porteurs de sens et leur rôle dans le discours est mis en valeur par cette recherche. La prosodie prend son sens autour de la notion d'informativité développée par l'auteur. Elle "marque ce qui est important pour le locuteur au moment de l'énonciation " (142). On va ici au-delà de la grammaire traditionnelle pour proposer une approche combinatoire alliant syntaxe, pragmatique et prosodie afin de rendre compte de la complexité et de la richesse du discours oral semi-spontané.

Dans l'avant-dernier chapitre de l'ouvrage, « Désaccentuation, composition, réduction, cliticisation: un continuum», Ruth Huart s'intéresse aux combinaisons de termes appartenant à des catégories grammaticales différentes et aux phénomènes phonétiques de réductions qui leur sont associées à partir de l'observation d'un corpus d'enregistrements et de débats tirés de la radio britannique sur une douzaine d'années. Les liens entre le choix d'écriture soudée et l'accentuation sont étudiés à la lumière des opérations énonciatives sous-jacentes. De la fusion graphique vers la cliticisation, l'auteur définit un continuum dynamique propre à la langue orale spontanée. L'auteur montre notamment que les formes verbales soudées (gonna, wanna, gotta), ne perdent pas seulement leur forme phonétique pleine mais aussi la «valeur modale » (159) qui était «le reflet du point de vue de l'énonciateur» (159). L'opposition entre forme soudée et formes distinctes ne représente pas une simple variante mais le choix d'une forme ou de l'autre permet au contraire de véhiculer des informations sur « l'intention de signifier de l'énonciateur » (164).

Il s'agit là d'un ouvrage d'une grande cohérence et d'une grande clarté. Les contributions sont riches et variées tout en gardant un même but : celui de montrer ou de nous éveiller à la complexité de l'oral (en particulier spontané), de dépasser les barrières de la grammaire prescriptive traditionnelle pour faire sens des «infractions » 
aux règles en combinant les approches, les disciplines, les niveaux d'analyse et de matérialité de la substance sonore. On s'éloigne des cloisons traditionnelles entre les disciplines pour se diriger vers une véritable approche dite intégrée. Cette approche permet de mettre en valeur les interfaces entre les différentes disciplines, méthodologies, et approches de la langue orale. On en reste intrigué, intéressé et nourri de pistes de recherche et de nouveaux questionnements. Cet ouvrage constitue un hommage réussi aux travaux fondateurs de Claude Charreyre, aux travaux riches et dynamiques des chercheurs du réseau OSLIA et à la dimension orale de la langue anglaise.

\section{INDEX}

Mots-clés : oralité, linguistique anglaise, énonciation, oral spontané, linguistique de corpus, prosodie, variations prosodiques, intonation, grammaire de l'oral, syntaxe, pragmatique, méthodologie, analyse de l'oral, analyse du discours

Keywords : English linguistics, spoken English, spontaneous speech, corpus linguistics, prosody, prosodic cues, intonation, grammar, syntax, pragmatics, discourse analysis, research methods

\section{AUTEURS}

SAANDIA ALI

Maître de conférences

Université de Toulouse - Jean Jaurès

saandia.ali@univ-tlse2.fr 